\title{
XXVII.
}

Aus der chirurgischen Abtheilung des Luisenhospitales zu Aachen

(Oberarzt Dr. W. Müller).

\section{Ueber einen ungewöhnlichen Ausgang der acuten Osteomyelitis.}

\author{
Von \\ Dr. A. Becker, \\ Assistenzarzt.
}

Wie das nach der ersten ätiologischen Klarstellung der acuten Osteomyelitis scheinbar abgerundete Bild derselben in neuerer Zeit mancherlei Erweiterungen erfahren musste, so kennen wir aus der neueren Litteratur auch bereits eine ganze Reihe von Typen im klinischen Bilde, welche von den früheren Beschreibungen mannigfach abweichen. Garré ${ }^{3}{ }^{*}$ ) hat im Jahre 1893, nachdem er bereits im 2 Jahre zuvor in der Festschrift für $\mathrm{K} o \mathrm{cher}^{4}$ ) dasselbe Thema behandelt hatte, zuerst ausführlich "besondere Formen und Folgezustände der acuten infectiösen Osteomyelitis" beschrieben und in zehn Abschnitten die selteneren Formen mit klinischen Beobachtungen aus der Tübinger chirurgischen Klinik belegt. Er zieht dabei besonders die tuberculöse Ostitis und die syphilitische Periostitis ossificans in differential-diagnostischer Hinsicht in Betracht.

Jedoch nicht nur zwischen Tuberculose oder Lues einerseits und Osteomyelitis anderseits sind diagnostische Zweifel möglich, sondern, was für den betreffenden Patienten viel folgenschwerer sein kann, auch die Differentialdiagnose zwischen Sarkom und Osteomyelitis ist unter Umständen ausserordentlich erschwert. So beschreibt Jakobsohn $\left.{ }^{6}\right)$ aus der Helferich'schen Klinik in Greifswald 2 Fälle von Sarkom des Oberschenkels, die unter dem Bilde der acuten Osteomyelitis verliefen, und erwähnt gleichzeitig noch fünf ähnliche Fälle aus der Litteratur. Umgekehrt beschreibt Kocher ${ }^{5}$ ) in den "Vor-

*) Die kleinen Ziffern im Text beziehen sich auf das am Schluss befindliche Litteraturverzeichniss. 
lesungen über chirurgisehe Infectionskrankheiten ${ }^{*} 2$ Fälle ron Osteomyelitis femoris, die sich unter dem Bilde eines Sarkoms entwickelt hatten, und reilht den ausführlichen Krankengeschichten noch einen dritten analogen, von Kummer und Tavel beobachteten Fall von Osteomyelitis tibiae an. Endlich beschreibt Jordan ${ }^{2}$ ) noch zwei ähnliche Fälle von Osteomyelitis humeri, bezw. femoris aus der Heidelberger chirurgischen Klinik, bei denen gleichfalls Anfangs die Diagnose auf Sarkom gestellt wurde. Auch möchte ich nicht die Arbeit von $\mathrm{Sw}$ entzitzki ${ }^{8}$ ) über atypische Formen von Osteomyelitis unerwähnt lassen; dieselbe ist mir jedoch nicht im Original, sondern nur nach dem Referat im Centralblatt für Chirurgie bekannt, wonach dieselhe „3 Krankengeschichten von jenen Fällen enthält, wo die Diagnose elıer auf Geschwulst (Sarkom), Syphilis u. s. w., als auf Osteomyelitis gestellt werden musste, und es sich doch nur um eigenthïmlich verlaufende Osteomyelitis handelte."

Das seltene Vorkommen von derartigen, Geschwülste vortäuschenden Bildungen bei der acuten Osteomyelitis - ich habe, wenn ich von der Swentzitzki'schen Arbeit absehe, nur obige 5 Fälle in der Litteratur finden können - veranlasste meinen Chef, Herm Dr. W. Mïller, im September vorigen Jahres auf der 71 . Versammlung. deutscher Naturforscher und Aerzte in München über drei von ilhm operirte Fälle zu berichten und eins der bei der Operation gewonnenen l'räparate zu demonstriren. Im Gegensatz zu jenen als Knochenauftreibungen von geschwulstförmigem Charakter imponirenden Bildungen handelt es sich in unseren Fällen um wesentliche Veränderungen von Weichtheilen, die auf dem Boden acuter Osteomyelitis entstanden sind und sowohl in pathologisch-anatomischer wie klinischdiagnostischer Beziehung eine atypisch verlaufende Osteomyelitisform darstellen, nach deren Beschreibung wir in der Litteratur vergeblich gesucht haben. Da diese 3 Fälle in mancher Bezieliung interessante Einzelheiten darbieten, so dürfte es sich auch in Rücksicht auf ihre Seltenheit, sowie die Bedeutung, welche sie in differential-diagnostischer Hinsicht haben, empfehlen, dieselben etwas ausführlicher zu beschreiben. Ich lasse zunächst die drei hierher gehörigen Beobachtungen folgen :

Fall 1. Frl. S. W. aus Haag, 18 Jahre alt, aufgenommen am 15. August 1896.

Patientin wird vom Arzte geschickt wegen einer Geschwulst am rechten Oberschenkel. Sie giebt an, dass diese Geschwulst seit 3 Jahren sich entwickelt habe und in letzter Zeit gewachsen sei. Patientin macht zugleich auf eine wallnussgrosse, ganz schmerzlose Geschwulst am linken 
Oberarme aufmerksam, die seit derselben Zeit bestehen soll. Sie giebt au, in letzter Zeit abgemagert zu sein.

Auf der Vorderfläche des oberen Drittels des rechten Oberschenkels befindet sich ein deutlich abgrenzbarer, annähernd halbkugeliger, gleichmässig derb sich anfühlender, mannsfaustgrosser Tumor, der dem Knochen zwar aufzusitzen scheint, gegen denselben aber deutlich verschieblich ist. Die Haut uber der Geschwulst zeigt normale Beschaffenheit und ist über derselben verschieblich.

Es wird die Diagnose gestellt auf Tumor, wahrscheinlich periostales Sarkom; Cyste, ev. Echinococcus nicht ausgeschlossen.

Am 16. August 1896 wird in Aethernarkose ein ca. $15 \mathrm{~cm}$ langer Exploratirschnitt uber die Höhe der Geschwulst angelegt. Nach Durchtrennung der Fascia lata und einer Schicht Muskeln stösst man auf ein knorpelhartes, weissliches, fibröses Gewebe, welches durchaus den Eindruck eines Fibroms macht und sich zum grössten Theil stumpf freilegen lässt unter Beiseitedrängung der grossen Gefässe. Auch jetzt noch ist die Geschwulst deutlich gegen den Knochen verschieblich. Um den Charakter des Tumors festzustellen, wird eine Längsincision in denselben gemacht. Dieselbe muss eine Gewebsschicht von reichlich $2 \mathrm{~cm}$ Dicke durchdringen, die nur als Fibrom aufgefasst werden kann. Erst durch die Eröffnung eines Hohlraumes im Centrum der Geschwulst klärt sich die Natur derselben auf. Die Höhle ist nämlich ein von weichem Granulationsgewebe und trübem Serum ansgefüllter Raum mit einem etwa $2 \mathrm{~cm}$ langen Corticalsequester von zackigem Aussehen. Von dem Hohlraum aus liess sich nach oben ein feinster Granulationsgang verfolgen, der zu einem Defect an der Vorderfäche des Femur an der Grenze von Schenkelhals und Schaft führte. Auskratzung desselben. Der Tumor wird zum grössten Theile exstirpirt. Drainage. Verband.

Die Diagnose wird nunmehr gestellt auf alte Osteomyelitis mit Bildung ungewöhnlich dicker, fibröser Schwarten um den Erkrankungsherd herum und durch die Züchtung des Staphylococcus pyogenes albus in Reincultur aus der trüben, serösen Flüssigkeit bestätigt.

Nachträglich erinnert Patientin sich auf Befragen, dass sie vor 5 Jahren an "Nervenfieber" erkrankt gewesen sei und damals mit heftigen Schmerzen im rechten Oberschenkel und rechtem Hüftgelenke längere Zeit zu Bette gelegen habe.

Der Verlauf ist ein normaler, so dass Patientin am 8. October 1896 geheilt nach Hause entlassen werden kann. Von einer Operation der kleinen Geschwulst am Oberarm wird, da seit langer Zeit dieselbe unverändert geblieben sein soll, einstweilen Abstand genommen.

Am 22. August 1899 kommt Patientin wieder zur Aufnahme. Sie will heirathen und bittet, vorber von der kleinen Geschwulst am linken Oberarme durch eine operative Entfernung derselben befreit zu werden. Dieselbe hat jetzt die Grösse eines Hühnereies, fuhlt sich sehr derb an und ist gegen den Knochen verschieblich. Die Haut ist liber der Geschwalst ein wenig entzündlich geröthet. Röntgenphotographie lässt den Knochen selbst intact erscheinen, ergiebt jedoch in den Weichtheilen im Bereiche des Tumors einen kleinen erbsengrossen Schatten, der vermuthlich einem Sequester entspricht. Entsprechend der Vorgeschichte wird 
die Diagnose auf Osteomyelitis humeri mit tumorartiger Schwielenbildung um einen Sequester herum gestellt. Bei der Operation am 4. November 1899 stellt sich die Geschwulst als ein durch Bindegewebe und derbere Schwiele abgekapselter Sack dar, der im Inneren weiche Granulationen und zwei erbsengrosse osteomyelitische Sequesterchen enthält. Da sich keine zum Knochen fübrende Fistel vorfindet, so wird der Knochen nicht weiter freigelegt, sondern nur die Abscesshöhle ausgekratzt und tamponirt. Wundverlauf normal. Patientin wird am 29. September 1899 nach Hause entlassen. Die Wunde ist bis anf eine kleine, nur noch wenig secernirende Fistel geheilt.

Am 13. December 1899 wird Patientin zum dritten Male aufgenommen, da die Fistel noch immer secernirt und keine Tendenz zur Heilung zeigt. Am 15. December wird in Narkose und Blutleere nochmals die Fistel gespalten und jetzt der Knochen freigelegt. Es zeigt sich, dass dicht unterhalb der oberen Epiphysenlinie des Humerus eine Fistel in den Knochen fuhrt. Der Humerus wird an dieser Stelle aufgemeisselt und ein ungefähr haselnussorosser Herd mit Sequester- und Granulationsbildung freigelegt. Säuberung desselben. Tamponade. Verband. Heilungsverlauf normal. Am 4. Februar 1900 wird Patintin mit normaler Function des Armes geheilt nach Hause entlassen.

Fall 2. Frl. Th. aus Aachen, 29 Jahre alt. Aufgenommen in die Privatklinik des Herrn Dr. M ttller an 13. März 1899.

Patientin stammt aus gesunder Familie und will frther stets gesund gewesen sein. Seit October 1898 verspurt Patientin geringe Schmerzen und ein Spannungsgefuhl in der rechten Kniekehle. Seit November 1898 muss Patientin fast ununterbrochen liegen, da die Spannung immer mehr zunimmt und in der Kniekehle eine Geschwulst sich bildet, die Patientin beim Gehen behindert. Die Geschwulst war bis März 1899 - Weihnachten 1898 batte Herr Dr. Muller die Patientin zum ersten Male gesehen - deutlich gewachsen. Die Schmerzen, welche Patientin im Knie verspurt, sind, abgesehen von dem Spannungsgefuhl, nur unerheblich, dagegen hinkt Patientin stark beim Gehen, will auch etwas abgemagert sein.

A ufnahmebefund: Kräftig gebaute, sonst gesunde Person in ziemlich gutem Ernährungszustande. In der rechten Kniekehle zwischen den Sehnen des Musc. biceps und semitendinosus fuhlt man einen die Form und Grösse eines längshalbirten Gänseeies darbietenden, gegen die Cmgebung scharf abgegrenzten, ziemlich derb sich anfuhlenden Tumor von glatter Oberfläche. Derselbe ist gegen die knöcherne Unterlage deutlich verschieblich. Die Haut ist nicht mit dem Tumor verwachsen und zeigt normale Beschaffenheit. Flexion im rechten Kniegelenke ist bis zu einem Winkel von $90^{\prime \prime}$ gut ausfithrbar. Das Gelenk selbst ist frei von Erguss und Schwellung. Fin Röntgenbild lässt keine pathologischen Veränderungen erkennen. Die Leistendrttsen sind nicht geschwollen, auch sonst sind keinerlei Metastasen nachweisbar. Temperatur normal. Die Diagnose wird auf Tumor der Kniekehle, wahrscheinlich von der Fascia poplitea ausgelsend, gestellt (Fibrom, Sarkom?) und Patientin dringend eine operative Entfernung derselben angerathen, in die sie auch einwilligt. 
14. März 1899 Operation in Aethernarkose. Es wird in Blutleere der Tumor durch einen ungefähr $10 \mathrm{~cm}$ langen, in der Mitte der Kniekehle längs verlaufenden Schnitt freigelegt. Es handelt sich um einen unter der subcutanen Fettschicht gelegenen, derben, fibrösen Tumor, der sich ohue wesentliche Schwierigkeiten, zum Theil stumpf, aus seiner Umgebung auslösen lässt. Nachdem derselbe bis auf einen etwa bleistiftdicken Stiel, welcher sich neben den Kniekehlengefässen in die Tiefe einsenkt, lospräparirt und abgetragen ist, zeigt sich hier eine Stelle des Tumor cystisch erweicht. Dieselbe wird eröffnet, und erweist sich als ein ungefähr haselnussgrosser Hohlraum, in dem sich ein zackiger typischer osteomyelistischer Corticalsequester von der Grösse einer Bohne findet, umgeben von geringen Mengen trübseröser Flüsigkeit und Granulationen. Der Stiel des Tumors, der einen feinsten Granulationsgang im Centrum hat, geht von hier aus zur äusseren nnd unteren Partie des Condylus internus femoris nahe dem Gelenkspalte. Drainage der Wundhöhle, Hantnaht, Verband. Normaler, fehlerfreier, aseptischer Wundverlauf, so dass Patientin am 25. März 1899 geheilt nach Hause entlassen werden kanu.

Bei der nachträglich nochmals anfgenommenen Anamnese erinnert Patientin sich, dass sie im Alter von 7 bis 8 Jahren - also vor ca. 22 Jahren! - von einer Fensterbank herunter gesprungen sei, wonach sie eine "Gelenkentztindung" des rechten Kniegelenkes bekommen habe. Sie habe damals 7 Monate lang mit "Kniegelenkentzindung" zu Bette gelegen und habe auch in den ersten Jahren nachher noch mitunter geringe Schmerzen im rechten Knie verspitrt.

Fall 3. Hüttenarbeiter J. N., 29 Jahre alt, aus Rötgen. Aufgenommen am 22. März 1899.

Patient will aus gesunder Familic stammen und fruher nie krank gewesen sein. Im Juli 1898 will Patient sich einen starken „Bluterguss ins linke Kniegelenk" zugezogen haben durch einen Sprung von einem Wagen. Patient hat damals mehrere Wochen gelegen und klagt seit dieser Zeit uber Schmerzen im ganzen linken Beine. Er hat nur mit Unterbrechungen seit dieser Zeit wieder arbeiten können. Seit 3 bis 4 Monaten bemerkt Patient eine zunchmende Anschwellung des linken Oberschenkels im mittleren Theil, die besonders in den letzten Wochen ganz erheblich stärker geworden ist. Da in Folge der immer grösser werdenden Spannung im Oberschenkel die Schmerzen geradezu unerträglich werden, so macht der behandelude Kassenarzt (vor 8 Tagen) eine kleine Incision in die Geschwulst, wonach die Schmerzen etwas geringer werden. Auf Veranlassung dieses Kassenarztes wird Patient auf der chirurgischen Abtheilung des Louisenhospitals aufgenommen. Er will in letzter Zeit unbedeutend abgemagert sein.

A ufnahmebefund: Kräftig gebauter junger Mann in gutem Ernährungszustande, mit mässig starkem Fettpolster und im Uebrigen gesunden Organen. Der linke Oberschenkel ist im Verhältniss zum rechten stark geschwollen und zwar ist in der Mitte des Oberschenkels die Schwellung am bedentendsten, um von hier aus nach dem Huft- wie Kniegelenke hin spindelförmig abzunebmen. Die Schwellung macht durchaus den Eindruck eines grossen, dem Knochen an der äussercn und 
vorderen Seite aufsitzenden, jedoch gegen denselben etwas verschieblichen soliden Tumors. Die Leistendritsen sind beiderseits geschwollen und hart anzufilhlen, links stärker als rechts. In der Mitte des Oberschenkels befindet sich eine $3 \mathrm{~cm}$ lange Incisionswunde. Durch dieselbe werden aus dem Tumor mehrere kleinere Stutckchen entfernt, die bei der mikroskopischen Untersuchung aus Rundzellen bestehen und die Annahme eines Sarkoms wohl rechtfertigen. Eine Röntgenaufnahme lässt keine pathologischen Veränderungen am Knochen erkennen. Demnach wird die Diagnose auf periostales Sarkom des Oberschenkels gestellt.

Am 23. März 1899 Operation in Aethernarkose und Blutleere. Es wird zunächst der Tumor durch grossen Schnitt vorn aussen freigelegt. Derselbe liegt subfascial und besteht aus fibrösem, theilweise sulzigem, theilweise schwieligem Gewebe von mindestens 4 bis $5 \mathrm{~cm}$ Dicke. Bei tieferem Eindringen in den Tumor wird ein kleiner IIohlranm eröffnet, aus dem ein Tröpfehen Eiter hervorquillt, dahinter befindet sich osteomyelitischer Sequester von etwa $1 \mathrm{~cm}$ Länge $1 / 2 \mathrm{~cm}$ Breite. Exstirpation des ganzen mannsfaustgrossen, schwartigen Tumors, was zum Theil stumpf gelingt. Es führt keine Fistel zum Knochen. Gegenöffnung an der lateralen Seite des Oberschenkels. Drainage. Hautnaht. Verband.

14. April 1899. Patient wird heute nach fieberfreicm aseptischem Verlaufe mit nur noch kleiner granulirenden Wunde zur ambulanten Nachbehandlung entlassen. Die Dritsenschwellung in der Leistengegend ist nahezu ganz zurïckgegangen.

Das Charakteristische an diesen 3 Fiillen berulit in dem anatotomischen Befunde und lïsst sich kurz ausdrïcken: eine an's Monströse srenzende, geschwulstförmige Schwielenbildung in Weichtheilen, veranlasst durch einen gewanderten kleinen, acut osteomyelitischen Scquester. Im Gegensatz zu den oben angeführten (Kocher, Jordan) als Knochensarkome imponirenden Osteomyelitis-Formen handelt es sich hier, wie erwähnt, um schwielige Tumoren, welche vielmehr als periostale resp. fasciale unschriebene Geschwülste erschienen. Die Annahme solcher war besonders gerechtfertigt durch die Beweglichkeit derselben gegen den Knochen, ilıre derbe, gleichmässige Beschaffenlıeit, das Fellen von Fluctuation. Dazu kam die zunächst irreführende Anamnese, die erst post operationem die wünschenswerthen Ergäinzungen gab. Ferner kam dazu in 2 Fällen das Fehlen von Sclımerz, die Anfangs scheinlsar ganz allmähliche Entwicklung; die erst in letzter Zeit einem raschen Wachsthum der Geschwulst Platz machte, die Abmagerung des Patienten. Das im 3. Falle voraufgegangene Trauma kann auch ebensowolı für Sarkom wie Osteomyelitis in ätiologischer Beziehung rerwerthet werden, da im Anschluss an ein Trauma wicderholt das Auftreten eines Sarkoms einwandfrei bcobachtet ist. In diesem letzten Falle wurde die Diagnose Sarkom ausserdem noch um so wahrscheinlicher, als die mit dem scharfen 
Löffel zuvor entfernten Tumormassen makroskopisch wie mikroskopisch sich mit der Annahme eines in Zerfall begriffenen Rundzellensarkoms wohl vereinigen liessen. Wenn auch bei der nach der Operation nochmals aufgenommenen Anamnese sich Anhaltspunkte fanden, welche das Voraufgehen einer acuten Osteomyelitis durchaus wahrscheinlich erscheinen liessen, so ist bei unseren Fällen doch zu betonen, dass nichts auf Abscess und Fistelbildungen weder früher noch jetzt deutete. Dazu kam in Fall 2 und 3 der negative Befund im Aktinogramm.

Bei der Operation selbst, die sich in allen 3 Fällen zunächst nur auf eine Freilegung des Tumors und Feststellung seines Charakters beschränken sollte, zeigte sich, dass sich die fraglichen Geschwülste jedes Mal mehr oder weniger leicht aus dem sie umgebenden Gewebe auslösen liessen, und dass auch während der Operation der Verdacht auf Tumor fortbestand. Während im 3. Falle kein Herd im Knochen mehr gefunden werden konnte, führte in den beiden anderen Fällen von diesem Erweichungsherd ein Gang zum Knochen hin.

Das im 2. Falle bei der Operation gewonnene Präparat veranschaulicht das Ungewöhnliche dieser Verhältnisse (dünngestielte Geschwulst) in besonders deutlicher Weise und gebe ich die Abbildung dieses Präparates nach einer photographischen Aufnahme in natürlicher Grösse:

In der Mitte des Tumors liegt in einem eröffneten Hohlraume $(\mathrm{H})$ der ungefähr bohnengrosse Corticalsequester (S). Von hier geht ein ungefähr $5 \mathrm{~cm}$ langer, bleistiftdicker Stiel (St) aus, der von einem feinsten Granulationsgange durchbohrt ist. Derselbe führt zum Condylus internus des Femurs hin.

Was ferner besonders interessant ist, so hat in den 4 Fällen, in welchen aus der Tumormasse Stückchen zum Zwecke der mikroskopischen Untersuchung entfernt wurden, jedes Mal das mikroskopisehe bild Sarkom, und zwar sowohl Spindelzellen- und Rundzellen-Sarkom wie Angiosarkom vorgetäuscht, obgleich es sich doch, wie der spätere Verlauf gezeigt hat, nur um entzïndliche Granulationsmassen gehandelt hat.

Einen vielleicht in die Kategorie unserer Fälle gehörigen Fall erwähnt $\mathrm{H}$ of meister ${ }^{9}$ ) in der Discussion, die sich dem dieses Thema behandelnden Vortrage meines Chefs auf der Münchener Naturforscherversammlung anschloss. In diesem von II of meister operirten Falle hatte sich im Anschluss an eine starke Contusion der Scapula allmählich ein faustgrosser Tumor entwickelt, der anfänglich für ein Gumma oder Sarkom gehalten wurde. Die Operation ergab einen 
bindegewebigen Tumor, der in die Musculatur eingewachsen war, und dessen mikroskopische Lntersuchung eine circumscripte Schwicle periostaler Natur erkennen liess.

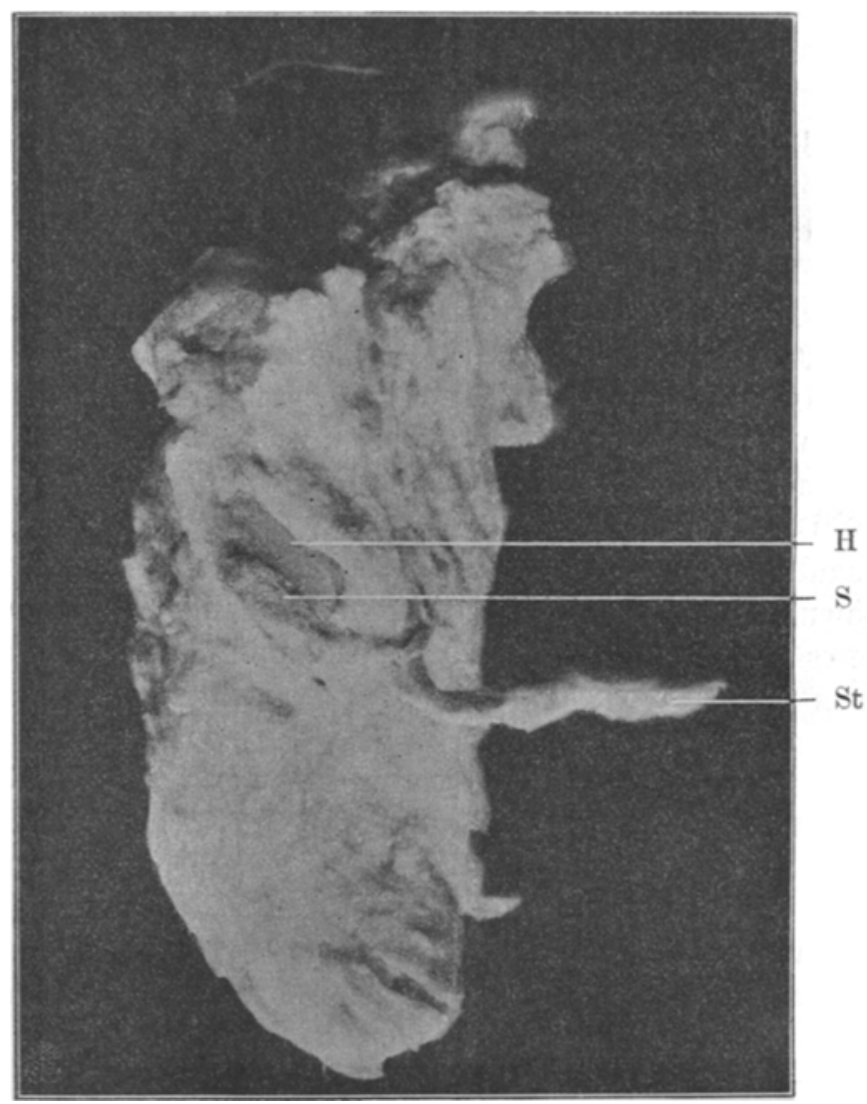

Was endlieh die Bedeutung solchen ungewöhnlichen Verlaufes der acuten Osteonyelitis anbetrifft, so ergiebt sich dieselbe aus der Verschiedenheit der Therapie und Prognose von Sarkom und Osteomyelitis von selbst. Jordan 2) mahnt dementsprechend mit Recht bei der Beurtheilung von Knochensarkomen zur Vorsicht und empfiehlit, grundsätzlich ror Vornahme einer verstümmelnden Operation zwecks sicherer Diagnosenstellung den Explorativschnitt auszuführen, was, wie auch unsere Fälle lehren, gewiss zu beherzigen ist. Der Befund von Sequestern und der bakteriologische Nachweis ron Staphylokokken sei dann ausschlaggebend für die Diagnose Osteomyelitis, während 
doch, wie wir oben gesehen haben, die mikroskopische Lntersuchung von excidirten Stïckchen des Tumors nicht immer vor Irrthümern in der Diagnosenstellung sehützt.

Ob die für solche Fälle charakteristische enorme Schwielenbildung auf Grund eines so geringfügigen Anreizes, wie ihn kleine Sequester doch darstellen, auf einer besonderen Disposition, etwa wie bei der Narbenkeloidbildung, beruht, ist schwer zu entscheiden, aber nicht unwahrscheinlich. Wenn man einen Zeitraum von 22 Jahren, wie in Fall 2, von dem Beginn der Erkrankung bis zur Bildung eines ausgesprochenen Tumors vor sich hat, so erscheint die massenhafte Production ron Schwiele (Fremdkörpertumor!) am Ende begreiflich, anders, wenn nur ein halbes Jahr für die Entwicklung solch monströser Schwielenbildung angegeben wird. Dass es sich übrigens in unseren Fällen um ein ganz anderes Bild gehandelt hat, als das, welches bei alten fistulösen Fällen von acuter Osteomyelitis, besonders am Oberschenkel, häufig ist, nämlich um schwielige diffuse Entartung der Musculatur, geht wohl aus der Schilderung der Fälle zur Genüge, hervor.

\section{Litteraturverzeichniss.}

1) Jorlan, Die acute Osteomyelitis und ihr Verhältniss zu den progenen Infectionen, auf Grund klinisch-bakteriologischer Beobachtungen, sowie des jetzigen Standes der Bakteriologie bearbeitet. Beitrüge z. klin. Chirurgie. 3id. X. Heft 3. S. 58 iff.

2) Jordan, Leber atypische Formen der acuten Osteomyelitis. Beiträge $z$. klin. Chirurgie. Bd. XV. Heft 2. S. $457 \mathrm{ff}$.

3) Garre, Cober besondere Formen und Folgezustände der acuten infectiöscn Osteomyelitis. Beitrüge z. klin. Chirurgie. Bd. X. Heft 2. S. $241 \mathrm{ff}$.

4) Derselbe. Einige seltene Erscheinungsformen der acuten infectiösen Osteomyelitis. Festschrift zu Ehren des Herrn Prof. Kocher zu Bern. Wiesbidlen, J. F. Bergmann. 1 s 91.

5) Kocher und Tavel, Vorlesungen über chirurgische Infectionskrankheiten. I. Theil. 1595 .

6) Jakobsohn, Ein Beitrag zur Kenntniss der Sarkome langer Röhrenknochen. Inaug.-Dissert. Greifswald 1595.

7) Mosheim, Ein Fall von periostalem Sarkom der Fibula. Inang.-Dissertation. Wïrzburg 1892.

s) W. A. Swentzitzki, Ueber atypische Formen von Osteomyelitis. (Aus der chirurgischen Facultätsklinik Prof. B obr of f's.) Die Chirurgie 159\%. Nr.9 (Russisch). Referirt: Centralblatt für Chirurgie. 1595. Nr. 6. S. 172.

9) Bericht über die 71 . Versammlung deutscher Naturforscher und Aerzte in München, vom 17.-22. September 1399. Referirt: Hïnchener medicinische Wochenschrift. 1599. Nr.40. S.1314. 